\title{
High-Efficiency Dual-Polarized Broadband Reflecting Metasurface Using Continuous Polarization Conversion Technique and Element with Multi Degree of Freedom
}

\author{
Majid Karimipour ( $\nabla$ m.karimipour@arakut.ac.ir) \\ Arak University of Technology \\ Iman Aryanian \\ Iran Telecommunication Research Center (ITRC)
}

\section{Research Article}

Keywords:

Posted Date: February 10th, 2022

DOI: https://doi.org/10.21203/rs.3.rs-1334587/v1

License: (c) (1) This work is licensed under a Creative Commons Attribution 4.0 International License. Read Full License 


\title{
High-Efficiency Dual-Polarized Broadband Reflecting Metasurface Using Continuous Polarization Conversion Technique and Element with Multi Degree of Freedom
}

\author{
Majid Karimipour ${ }^{1, *}$ and Iman Aryanian ${ }^{2}$ \\ ${ }^{1}$ Arak University of Technology, department of electrical engineering, Arak, +98 , Iran \\ ${ }^{2}$ Iran Telecommunication Research Center (ITRC), Tehran, +98, Iran \\ *m.karimipour@arakut.ac.ir
}

\begin{abstract}
In this study, two effective approaches are combined which are implemented at the element design level and system design level to simultaneously improve the frequency bandwidth and aperture efficiency of a dual-polarized single-layer reflecting metasurface. At the element design level, a broadband behavior is realized by using the polarization conversion technique (PCT) which is a novel technique to enhance the bandwidth of the element. To this end, an anisotropic metasurface with I-shaped metal patch is proposed for rotating the polarization of the wave emitted from a point source by $90^{\circ}$ and making a continuous phase shift in a full range of $360^{\circ}$ within $8-18 \mathrm{GHz}$. Therefore, a completely equiphase aperture is achieved leading to enhancing the metasurface performance such as directivity and aperture efficiency and reducing the sidelobe level compared to reflecting metasurface developed by 1-bit phase quantization technique. At the system design level, the three-frequency phase synthesis (TFPS) method, which is based on determining the best constant reference phase for the aperture, is used and the corresponding constant reference phases are optimized to minimize the phase error in the whole band. The combination of TFPS and PCT enhances the effectiveness of the TFPS method considerably. An 841 element reflecting metasurface with aperture dimensions of $290 \mathrm{~cm} \times 290 \mathrm{~cm}$ is designed, simulated, and fabricated in Ku-band to verify the concept. The measurement results show that the 1-dB gain bandwidth before and after combining PCT and TFPS techniques are 17.47\% (14.1 GHz to $16.8 \mathrm{GHz}$ ) and $30.3 \%(14 \mathrm{GHz}$ to $19 \mathrm{GHz}$ ), respectively. In addition, the maximum aperture efficiency of the proposed metasurface is $63.62 \%$ which occurs at $14.5 \mathrm{GHz}$.
\end{abstract}

\section{Introduction}

Reflecting metasurface (or reflectarray) is a good candidate for high gain applications such as satellite communication. This is why it benefits from the advantages of reflector and classic array structures ${ }^{1}$. In general, a reflecting metasurface manipulates the phase of the wave impinging from the feed and reflects it with the desired characteristic such as direction, shape, polarization, and beamwidth ${ }^{2-5}$. This is done by introducing a given progressive phase shift over the reflected wave by designing a proper phase shifting surface or making an artificial impedance surface to engineer the reflected waves ${ }^{6}$. Although the metasurface has many advantages, it suffers from narrowband behavior. Frequency bandwidth enhancement has always attracted much attention by the communication engineers. So far, several efforts have been done to broaden the bandwidth of reflecting metasurface. All these methods can be divided into two branches: (I) broadening approaches at the element design level and (II) broadening approaches at the system design level.

Broadband methods at the system design level refer to those techniques that deal with the metasurface configuration neglecting the element type, such as the phase synthesis methods. For instance, the authors in Ref. ${ }^{7}$ introduce a broadband method at the system design level based on double frequency phase shift (DFPS) synthesis and an optimization routine is adopted to find the optimum constant reference phase at two-interval frequencies of the working band. It can be simply inferred that the DFPS technique has the great potential to enhance the bandwidth neglecting the element type. For instance, a $16.7 \%$ of $1.5-\mathrm{dB}$ gain bandwidth is achieved by a simple phasing element with a single-layered configuration ${ }^{7}$. Therefore, it is found that the effectiveness of the the phase synthesis method can be considerably enhanced by using wideband phasing elements. For example, exploiting this fact, a broadband reflecting metasurface with a 1-dB gain bandwidth of $21 \%$ was developed $^{8}$.

At the element design level, several broadband techniques are introduced in the literature to improve the bandwidth behavior of phasing elements in reflecting metasurfaces ${ }^{9-16}$. Recently, a novel broadband technique is introduced which is based on the polarization rotation of the impinging waves from the feed which causes to working of the metasurface in cross-polarization 


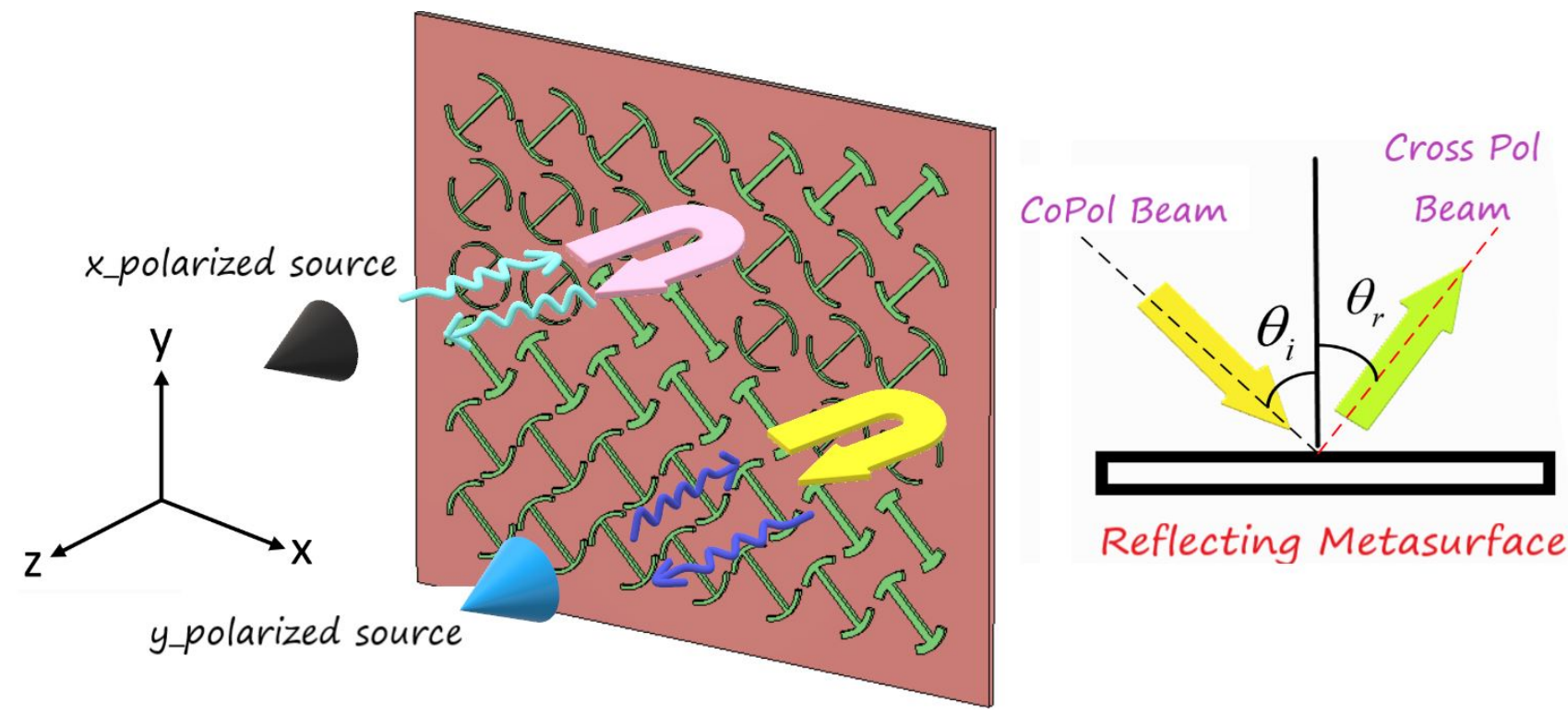

Figure 1. The proposed wideband reflecting metasurface working in cross-polarization mode when illuminated by a point source.

mode ${ }^{17}$. This technique, along with the studies that followed (Ref. ${ }^{18}$ and Ref. ${ }^{19}$ ) improves the reflecting metasurface bandwidth up to $20 \%$ by only the use of single-layered configurations. In this regard, several works are reported to design of wideband or multi-band polarization conversion metasurfaces ${ }^{20-24}$. It can be understood that there is more freedom to manipulate the phase of reflected waves in cross-polarization mode compared with the co-polarization (copol) one in reflective structures ${ }^{17,19,25}$. For example, the authors in Ref. ${ }^{25}$ exploit this interesting feature to design an ultra-thin transmit-reflecting metasurface structure. Similarly, the authors in Ref. ${ }^{17}$ developed a 1-bit geometrical spatial phase shifter to rotate the polarization of the reflected wave. Although the 1-bit reflecting metasurface has simple architecture compared with a completely equiphase aperture, the radiation performance such as the aperture efficiency of the radiator is relatively poor in these structures. For instance, it can be inferred from Ref. ${ }^{17}$ that the imperfect beam collimation in 1-bit reflecting metasurface causes to $3.8 \mathrm{~dB}$ gain reduction in the pattern compared with the beams constructed by continuous phasing aperture. Furthermore, sidelobe level enhancement is inevitable in 1-bit phase quantization apertures specifically in the off-centered feed configuration. To circumvent this drawback, a polarization conversion unit cell with the capability of generating continuous phase shifting over the reflected wave was introduced in which the $1-\mathrm{dB}$ gain bandwidth up to $18.5 \%$ was reported using this technique ${ }^{19}$.

To further improve the bandwidth of reflecting metasurface and achieve high aperture efficiency, at the same time, two broadening approaches introduced in Ref. ${ }^{7}$ and Ref. ${ }^{17}$ are effectively combined and modified in this paper. As stated before, these two approaches are implemented at the element design level and system design level. At the element design level, a single layer I-shape element with its mirror counterpart is employed to not only cover a full cycle of $360^{\circ}$ but also has an identical behavior in the reflection phase for the cross-polarization mode within 8 to $21 \mathrm{GHz}$. It is realized by adopting the multi-degree of freedom (MDoF) technology in the design of the element. Thanks to the use of lossless metasurface with reciprocal elements in the structure, time-reversal symmetry is maintained, which ensures that the metasurface shows a similar response for both yand x-polarized impinging wave and accordingly co and cross-polarization reflection coefficients are similar for both cases. 


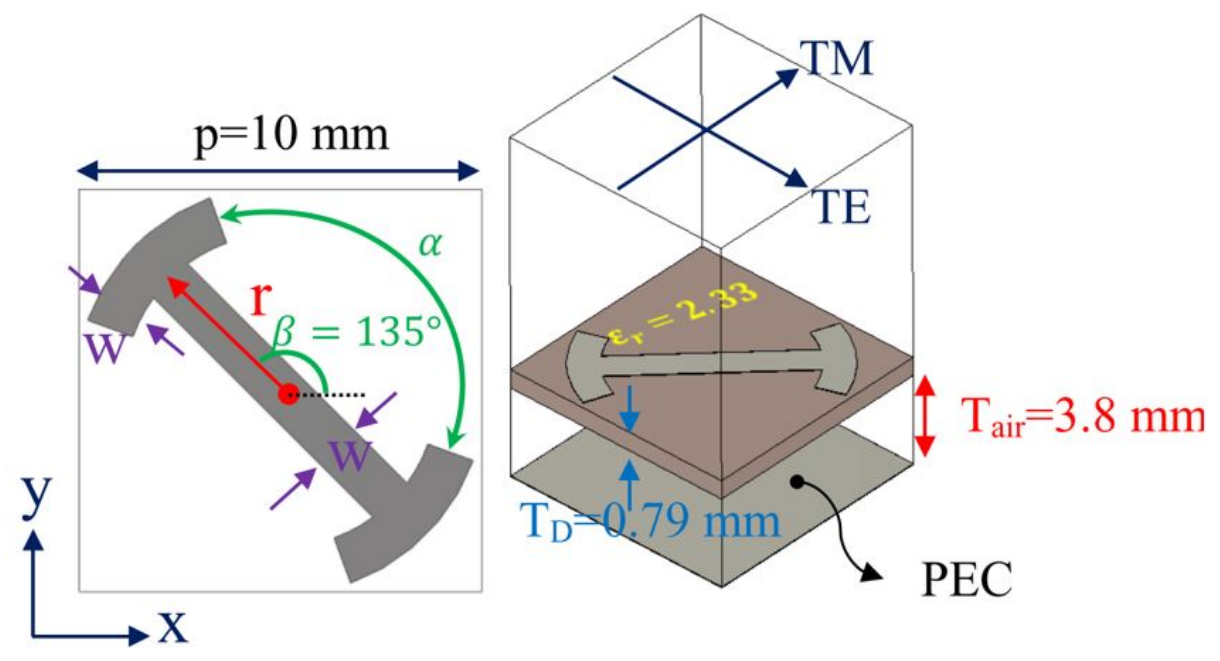

Figure 2. Polarization rotating element and its geometrical parameters.

Figure 1 depicts the working mechanism of the proposed reflecting metasurface in cross-polarization mode. It is worth noting that combining the method introduced in ${ }^{7}$ and ${ }^{17}$ has a great advantage because it is found that the effectiveness of the broadband technique introduced in Ref. ${ }^{17}$ can further be improved if a broadband unit cell is utilized at the element design level.

\section{Element Design for polarization conversion}

In order to control the electromagnetic characteristics of the reflected cross-polarization wave from the metasurface in a wide frequency range to generate a versatile radiation pattern and emulate the behavior of classical antennas, a continuous phasing aperture should be developed which would be similar behavior in the frequency band of interest. The aim is to design an element working in cross-polarization mode and cover the Ku frequency band. Theoretical investigations indicate that the main feature of a reflective linear polarization converter is that it reflects two $\mathrm{x}$-and y-polarized electric fields with the same amplitude and $180^{\circ}$ phase deference with respect to the illuminated wave components. Apparently, it can be deduced that the metasurface should be selected from anisotropic structures with negligible loss to keep the amplitude of the reflected wave equal to the illuminated one. The working principle of the linear polarization conversion can be interpreted by introducing an additional coordinate system with two perpendicular axes which are rotated by $45^{\circ}$ with respect to the $\mathrm{XY}$ coordinate system. Detail discussion about the theory is presented in Ref. ${ }^{24}$.

In order to satisfy the cross-polarization conversion criteria, a simple I-shape with two additional arc stubs is used as the metal patch of the unit cell. As shown in Fig. 2, the metal patch is rotated by $45^{\circ}$ with respect to the $x$-axis to exhibit anisotropic behavior. The unit cell dimension of the proposed single layer element is $10 \mathrm{~mm} \times 10 \mathrm{~mm}$. The proposed element and its mirror have enough freedom to make a similar phase shift over the cross-polarization wave in the whole band. As can be observed in Fig. 2, the I-shaped metal patch is placed on a single layer dielectric whose thickness and dielectric constant are $0.79 \mathrm{~mm}$ and 2.33 , respectively. A gap with a given thickness of $3.8 \mathrm{~mm}$ is considered between the ground plane and dielectric to further enhance the bandwidth. The phase of the reflected cross-polarization wave changes dominantly by varying the $\alpha$ parameter as the first degree of freedom (FDF). It can simply be shown that the phase behavior of the element cannot meet the expectations and the element has not similar phase behavior in a wide frequency range by only the use of FDF technology. Therefore, the MDoF technology is adopted to meet the expectations. Doing so, a full parametric study is accomplished by CST software. It is found that three geometrical parameters, $w, r$, and $\alpha$, should be changed at the same time to observe wideband behavior for the element in cross-polarization mode. Figure 3 shows the optimum relationship between $w, r, \alpha$, when $\alpha$ is changed from $10^{\circ}$ to $160^{\circ}$ to guarantee the wideband behavior of the unit cell. In order to rotate the reflected polarization wave by $90^{\circ}$ with respect to that of illuminated from the feed, the amount of $\beta$ parameter should be selected either $45^{\circ}$ or $135^{\circ}$. This ensures the anisotropic behavior of the element. Simulations show that if $\beta$ is selected equal to $45^{\circ}$, all combinations of $w, r, \alpha$ provide 
only half cycle of the required phase in Ku-band, that is $180^{\circ}$ in cross-polarization mode. Therefore, a full cycle of $360^{\circ}$ for the reflecting phase, which is required to implement a completely equiphase aperture in cross-polarization mode, can easily be realized by selecting $\beta=45^{\circ}$ and $135^{\circ}$.

In light of the above discussion when the $\alpha$ parameter changes, other geometrical parameters $(w$ and $r$ ) should be selected from Fig. 3. Cross-polarization conversion behavior of the I-shaped element for various values of $\alpha$ is depicted in Fig. 4 when the y-polarized wave is assumed as the impinging wave. It is shown that the $S_{y y}$ is smaller than - $15 \mathrm{~dB}$ for all values of $\alpha$ indicating good polarization conversion performance. Three resonance frequencies are observed in the frequency band which varies with $\alpha$ parameter. Undoubtedly, the intense induced current distribution is formed on the metallic patch at these resonance frequencies.

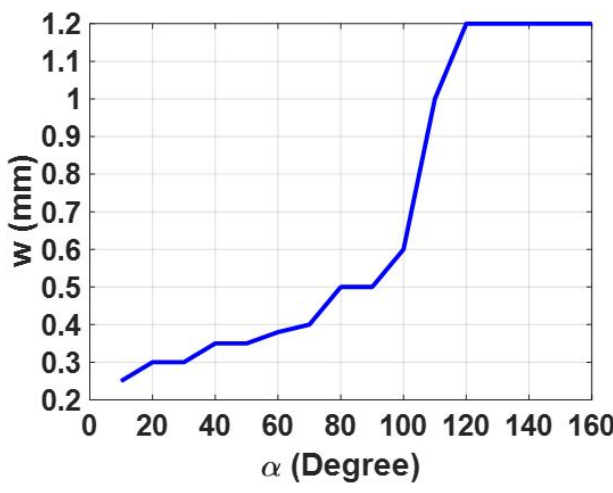

(a)

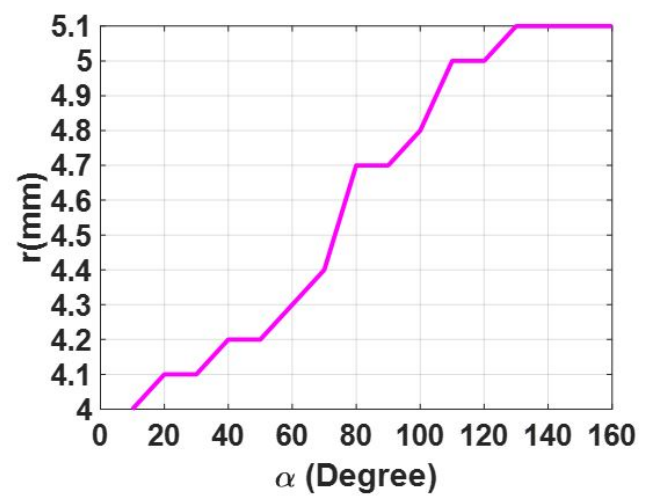

(b)

Figure 3. Optimum relation between the design parameters of the unit cell for realizing the wideband behavior in cross-polarization mode. (a) Optimum variations of $w$ when $\alpha$ is varied. (b) Optimum variations of $r$ when $\alpha$ is varied.

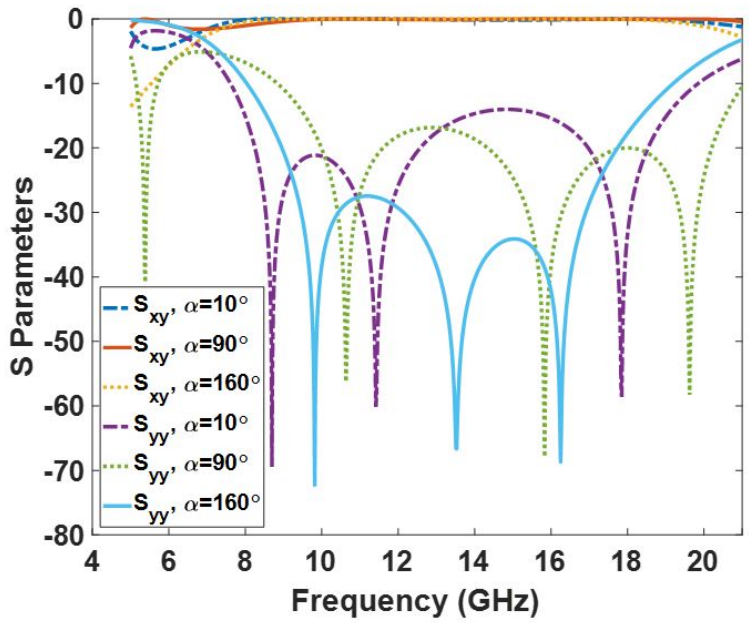

Figure 4. Co- and Cross-polarization conversion coefficients of the element for various values of $\alpha$. In this plot $\beta=45^{\circ}$. The parameters $w$ and $r$ are determined from Fig. 3

Reflection phase of cross-polarization wave with various values of $\alpha$ are shown in Fig. 5 for $\beta=-45^{\circ}$. It can be seen from Fig. 5a that the reflection phase of the cross-polarization component has a similar behavior from 8 to $21 \mathrm{GHz}$; however, the maximum coverage of the phase limits to $180^{\circ}$. Because of symmetry, its mirror (with $\beta=45^{\circ}$ ) has similar behavior in whole the band which has $180^{\circ}$ phase difference as shown in Fig. 6 . To complete the discussion, the effect of the incident angle of the wave is also examined over the cross-polarization reflection phase of the element within the band. Figure 7 shows the phase of 


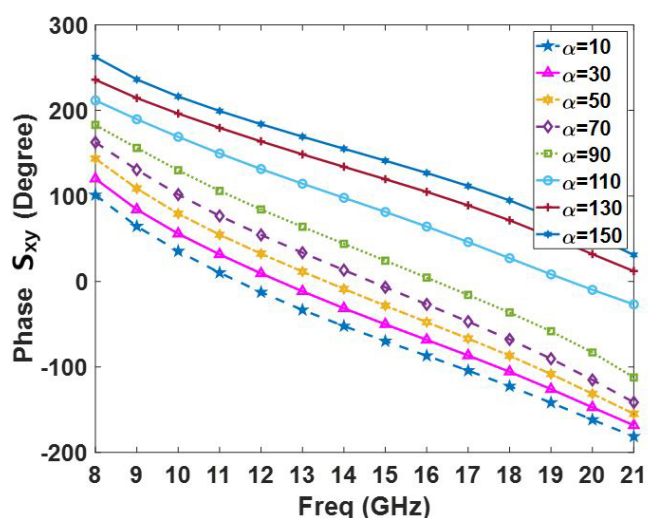

(a)

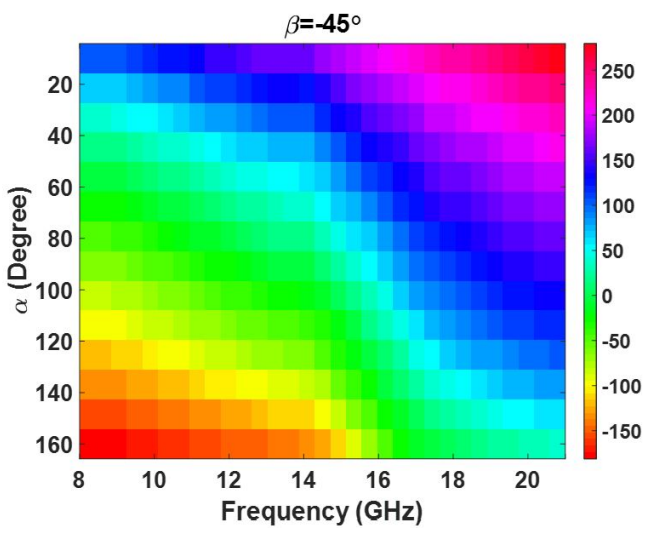

(b)

Figure 5. (a) and (b) Cross-polarization election phase behavior of the unit cell versus the frequency for several valuse of $\alpha$.

$S_{x y}$ for various values of angle of incidence. As can be seen in Fig. 7, the reflection phase behavior is nearly independent of the incident angle of the wave from 7 to $18 \mathrm{GHz}$. Although, the reflecting phase of the cross-polarization component becomes deteriorates beyond the $18 \mathrm{GHz}$ leading to a decrease in the antenna performance. It should be noted that a small number of elements placed near the edge of the metasurface are subjected to waves with high angles of incidence, so the undesirable effect of this phase degradation is mainly observed in the aperture efficiency and has a negligible effect on the shape of the reflected beam. This phenomenon is experimentally shown in the measurement results where the gain of reflected wave dramatically decreased beyond the $19 \mathrm{GHz}$.

Following the guideline described above, an equiphase aperture can be implemented by arranging various elements with independent geometrical parameters. The key factor to select the geometrical parameter associated with each element is its required reflection phase which is dictated by Snell's law for that position. Accordingly, when the required phase distribution is determined by Snell's law to guide the reflection beam at a given direction, $\alpha$ is determined and, in the next step, $w$ and $r$ will be determined from Fig. 3.

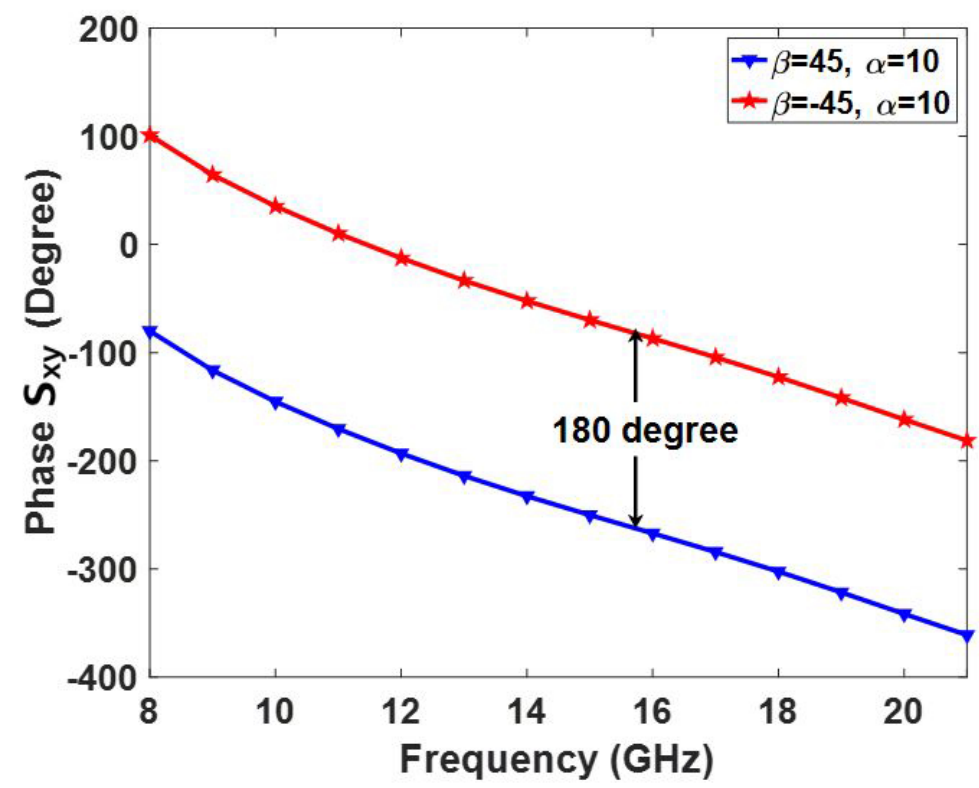

Figure 6. Reflection phase of $S_{x y}$ for $\beta=45^{\circ},-45^{\circ}$. The $\alpha$ parameter is considered to be $10^{\circ}$. 


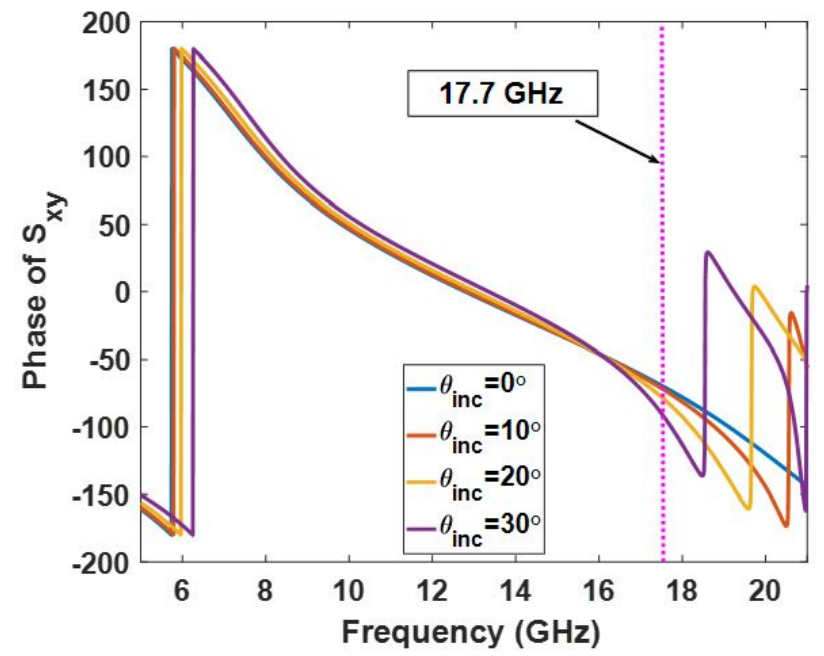

Figure 7. Reflection phase of $S_{x y}$ for various incident angle of the wave illuminated from the feed.

\section{Reflecting metasurface design by combining TFPS method and PCT}

Before explaining about TFPS method and how it can be combined with PCT to considerably enhance the frequency bandwidth of reflecting metasurface, some considerations need to be expressed. We know that anomalous reflection occurs near the interface of the metasurface and can be described by generalized Snell's law which relates the incidence angle of the wave, anomalous reflection direction, and the local phase gradient to each other ${ }^{26}$. In other words, for a beam reflected at a given direction, the desired phase distribution on the reflecting metasurface is dictated by Snell's law.

In general, all phases associated with all unit cells can be added or subtracted by a given constant value which is named the constant reference phase. The value of the constant reference phase is not important in the single frequency synthesis approach. However, in dual- or multi-frequency synthesis approach, the use of different constant reference phases results in a great variations in the electromagnetic features of the metasurface such as the realized gain. Therefore, the determination process of these constant reference phases can be done intelligently to enhance the bandwidth. The constant reference phases can effectively be determined during an optimization process. Doing that, a cost function is defined so that the phase error at two or more separate frequencies becomes minimum for all elements. This ensures that the final optimized phase distribution, which would be provided by the elements, has small variations when the frequency changes. We employ this approach at three frequencies in this work which is named TFPS. In this regard, the required phase, phase error, and the cost function are all represented in (1), (2), and (3).

$$
\begin{aligned}
& \phi_{m n, r}\left(f_{i}, \Delta \phi_{i}\right)=k_{i} \cdot\left(\left|\bar{r}_{m n}-\bar{r}_{f}\right|-\hat{u}_{0} \cdot \bar{r}_{m n}\right)+\Delta \phi_{i} \\
& P E_{m n}\left(f_{i}, \Delta \phi_{i}\right)=\left|\phi_{m n, a}\left(f_{i}, \Delta \phi_{i}\right)-\phi_{m n, r}\left(f_{i}, \Delta \phi_{i}\right)\right| \\
& C F\left(f_{i}, \Delta \phi_{i}\right)=\sum_{m, n}\left[\sum_{i} w_{i, m n} P E_{m n}\left(f_{i}, \Delta \phi_{i}\right)\right]
\end{aligned}
$$

In the above equations, the $i$ parameters are selected 1,2,3, for TFPS. The quantities $\phi_{m n, r}$ and $\phi_{m n, a}$ are the required and achievable phases for the element $m n$, respectively. The quantity $P E_{m n}$ denotes the phase error of the element $m n$. The weighting coefficients $w_{i, m n}$ are determined based on the illumination intensity over the elements on the metasurface. These coefficients are defined based on the point source characteristics and its relative position with respect to the metasurface. These coefficients are defined for every element at a specific frequency. In this work, particle swarm optimization (PSO) as a global search algorithm is employed to minimize the cost function. The role of the element and its phase-shifting behavior versus the frequency is observed in (2) which can be considerably decreased the phase error if a wideband unit cell is designed for this purpose. Investigations show that the choice of three frequencies, $f_{1}, f_{2}$, and $f_{3}$, can directly affect the metasurface bandwidth and, hence, the best choice is obtained after several repetitions of the algorithm for various values of frequencies in the band. In order to accelerate this process, we used the 2D-FFT algorithm to characterize the radiated 
beam for every phase distribution. That is, for every three frequencies, the optimum phase distribution for the aperture is obtained by the proposed algorithm, afterwards, the radiated beam is calculated by 2D-FFT and evaluate the 1-dB gain bandwidth, immediately. Finally, it is found that the best performance of the TFPS technique is achieved by choosing $f_{1}=$ 14.5 GHz, $f_{2}=17 \mathrm{GHz}$ and $f_{3}=19 \mathrm{GHz}$ and the corresponding optimum constant reference phases $\Delta \phi_{1}, \Delta \phi_{2}$, and $\Delta \phi_{3}$ are obtained equal to $47^{\circ}, 223^{\circ}$, and $80^{\circ}$, associated with $f_{1}, f_{2}$, and $f_{3}$, respectively, which are all extracted from the PSO algorithm.

\section{Feed horn description}

In a practical case, it is needed to use a point source to illuminate the metasurface. The best choice is to use the horn antenna whose phase center has minimum variations versus the frequency. This ensures that the feed has the minimum undesirable effect on the metasurface and, hence, the best performance of the metasurface is achievable. Since the traditional horn antennas experience great variations in their phase center positions when the frequency changes, a tangential profiled smooth-wall horn is designed to feed the metasurface so that its phase center variation is negligible from 10 to $18 \mathrm{GHz}$. A wideband coaxial-to-waveguide transition is also designed to excite the horn. The optimum dimension of the horn and its transition section are presented in Fig. 8 and Table. 1. The electromagnetic characteristics of the horn including reflection co-efficient realized gain, and the phase center position with respect to the front of the horn aperture are all shown in Fig. 9. It can be seen that the antenna gain varies from 13.8 to $15.1 \mathrm{dBi}$ in the frequency band of 9-18 $\mathrm{GHz}$. In addition, the variations of the phase center position limits to $4 \mathrm{~mm}$ when the frequency changes from $12 \mathrm{GHz}$ to $18 \mathrm{GHz}$, which is a very good achievement for illuminating the metasurface. This fact ultimately causes to assume that the focal-to-diameter ratio (f/D) is nearly fixed and consequently, the contribution of phase variation on the aperture of the metasurface imposed by the point source (or horn) would be small when the frequency is chaned.

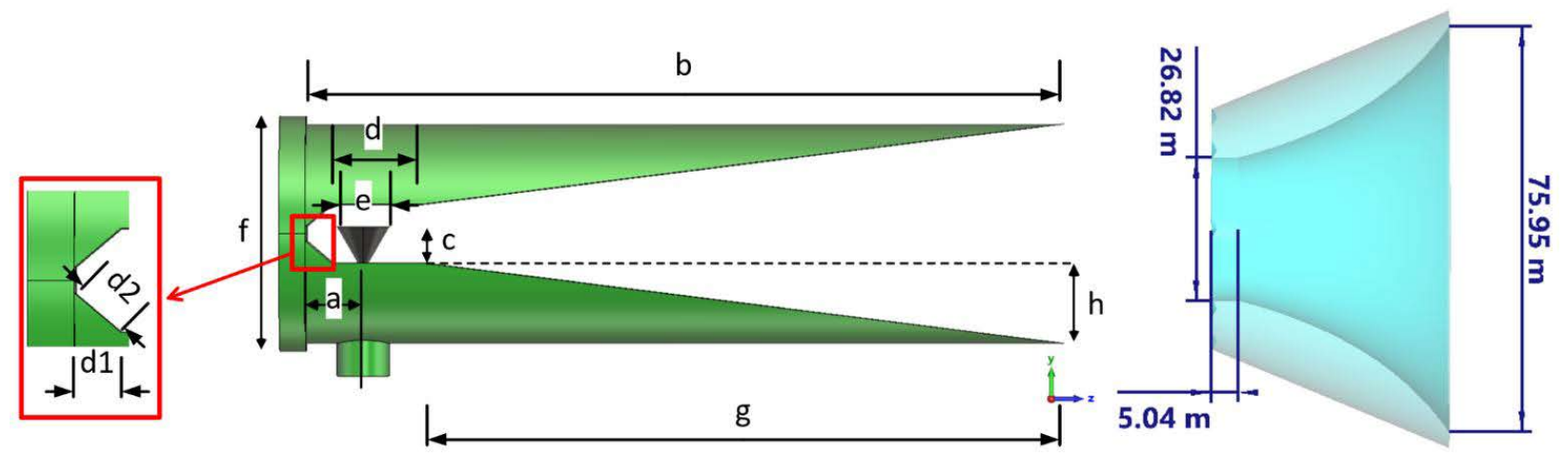

Figure 8. Antenna feed along with its wideband adapter. The conical waveguide section of the horn is not shown in this figure to better represent the transition section.

\begin{tabular}{|c|c|c|c|c|c|c|c|c|c|c|}
\hline Parameter name & $\mathrm{a}$ & $\mathrm{b}$ & $\mathrm{c}$ & $\mathrm{d}$ & $\mathrm{e}$ & $\mathrm{f}$ & $\mathrm{g}$ & $\mathrm{h}$ & $\mathrm{d} 1$ & $\mathrm{~d} 2$ \\
\hline \hline Value $[\mathrm{mm}]$ & 6.5 & 86.5 & 4.1 & 10.3 & 6 & 26.82 & 73.2 & 9.2 & 3 & 3.9 \\
\hline
\end{tabular}

Table 1. Optimum values of design parameters of the wideband coaxial-to-waveguide transition.

\section{Fabricated Prototype and Measured results}

In order to show the effectiveness of combining two aforementioned broadening techniques at system and element design levels, a representative metasurface is designed, simulated, and fabricated in Ku-band. Doing so, a $29 \mathrm{~cm} \times 29 \mathrm{~cm}$ aperture including 841 phasing elements is constructed which is illuminated by a feeding horn whose phase center is located ( $84 \mathrm{~mm}$, 0 , $232 \mathrm{~mm}$ ) and is pointed to the center of the metasurface aperture. The feed pattern is modeled by $\cos ^{q} \theta$ function with $q=6.5$ $(f / D=0.85)$. In order to decrease the feed blockage effect, the feed horn antenna is offset $20^{\circ}$ from the boresight direction, and the reflected main beam is designed at $20^{\circ}$. Following the design process mentioned above, the final arrangement of the array elements is obtained which is demonstrated in Fig. 10. 


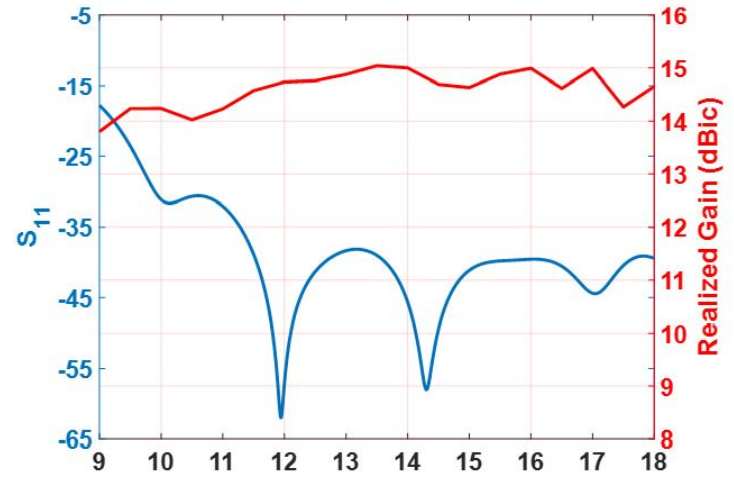

(a)

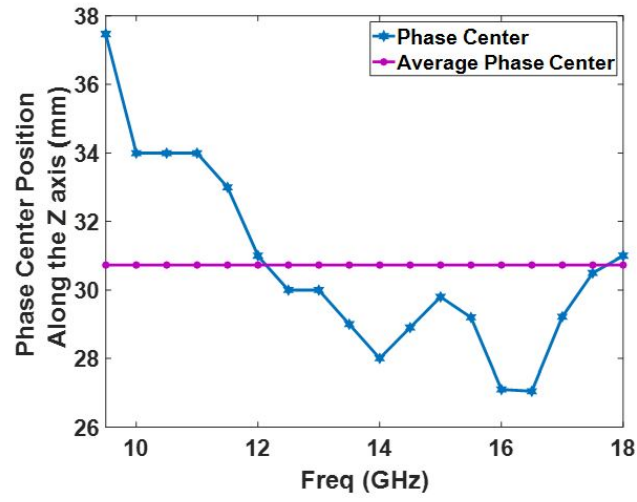

(b)

Figure 9. (a) Realized gain along with $S_{11}$ parameter of the horn antenna. (b) Phase center variations versus the frequency and its average.

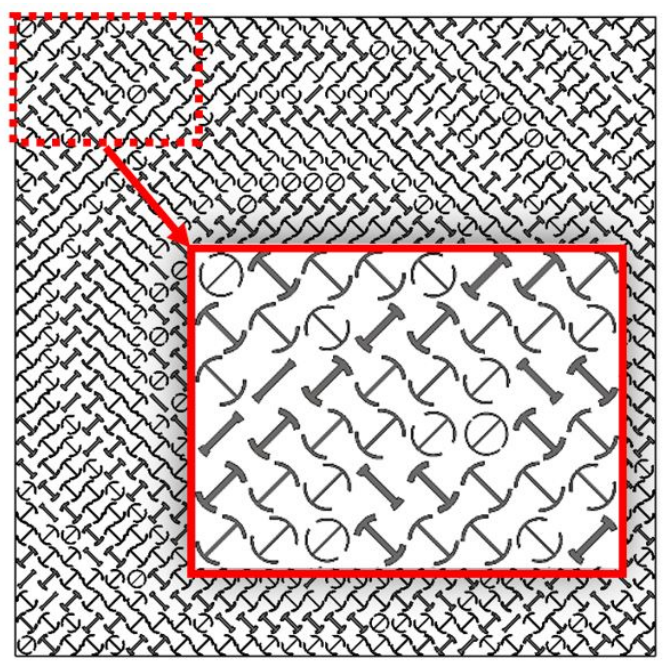

Figure 10. Arrangement of the elements obtained by combining the TFPS method and PCT.

The metasurface along with the feed horn is simulated in CST software with the time domain (TD) solver. The simulation was carried out on a PC using an Intel Core i $3.8 \mathrm{GHz}$ processor with 64 GB RAM and 1 TB SSD. Lossy copper (copper, annealed) is used as a metal. In addition, the open (add space) option is selected for the boundary condition in all directions and a waveguide port is used as the feeding. The three-dimensional (3D) demonstration of the patterns of the metasurface which is designed by the PRT+TFPS method is shown in Fig. 11 at three frequencies of $14 \mathrm{GHz}, 17 \mathrm{GHz}$, and $19 \mathrm{GHz}$. As can be shown in Fig. 11, the general shape of the 3D patterns is acceptable especially in the back-lobe region indicating the correct position of the phase center of the horn with respect to the metasurface and minimum phase error at these three frequencies. Additionally, the main beams are appropriately aligned to the predefined direction which shows that the progressive phase on the metasurface is calculated accurately in the whole band based on the TFPS method. Furthermore, the perfect beam collimation ensures the high aperture efficiency for the metasurface.

A prototype of the designed conical feed horn is shown in Fig. 12 which is fabricated by a machine controlled by computer numerically (CNC) with high accuracy. The waveguide and flare section of the antenna is fabricated as one unit and the transition section is fabricated separately which are assembled with some screws at the bottom. The fabricated metasurface is shown in Fig. 13 which is composed of a single copper cladding printed circuit board (PCB) using RT/duroid 5870 laminate and an aluminum metal plate as the ground. In order to keep the distance between the ground plate and dielectric layer, some spacers made up of plexiglass are placed between the two layers and are fixed by plastic screws (see the inset of Fig. 13). As 


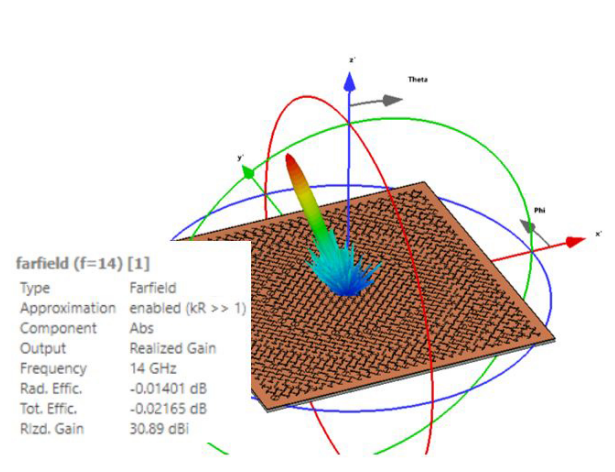

(a)

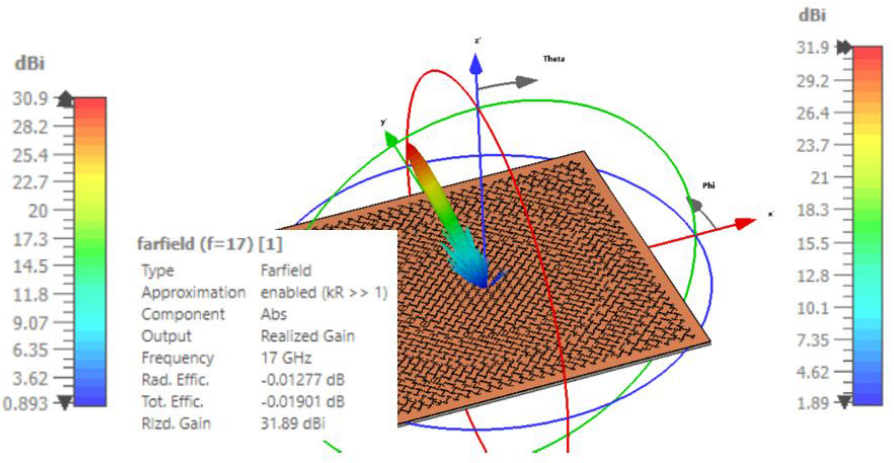

(b)

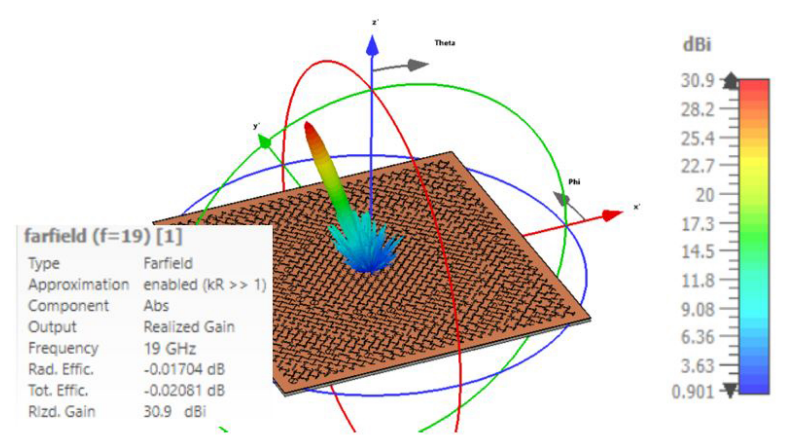

(c)

Figure 11. Three-dimensional demonstration of the pattern designed by PRT+TFPS method. (a) $\mathrm{f}=14 \mathrm{GHz}$. (b) f=17 GHz. (c) $19 \mathrm{GHz}$.

can be shown in Fig. 13, a wooden stand is fabricated to hold the horn antenna in front of the metasurface with $20^{\circ}$ offset.
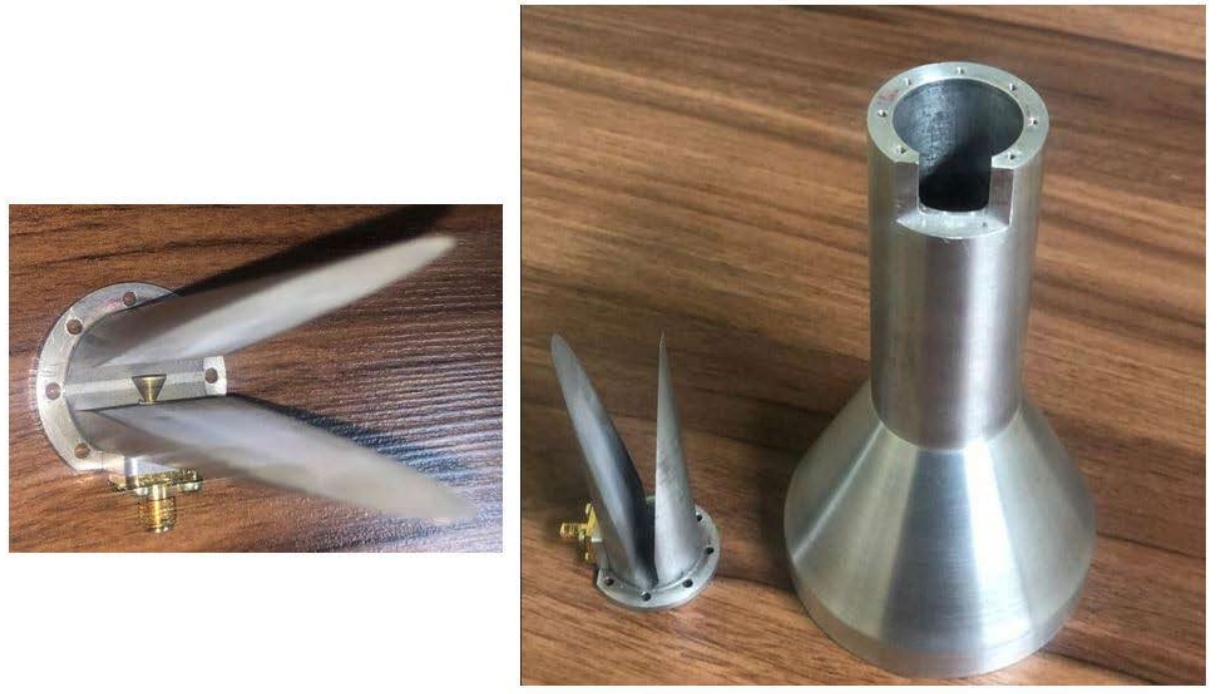

Figure 12. Fabricated horn antenna along with its wideband coaxial to waveguide transition. 


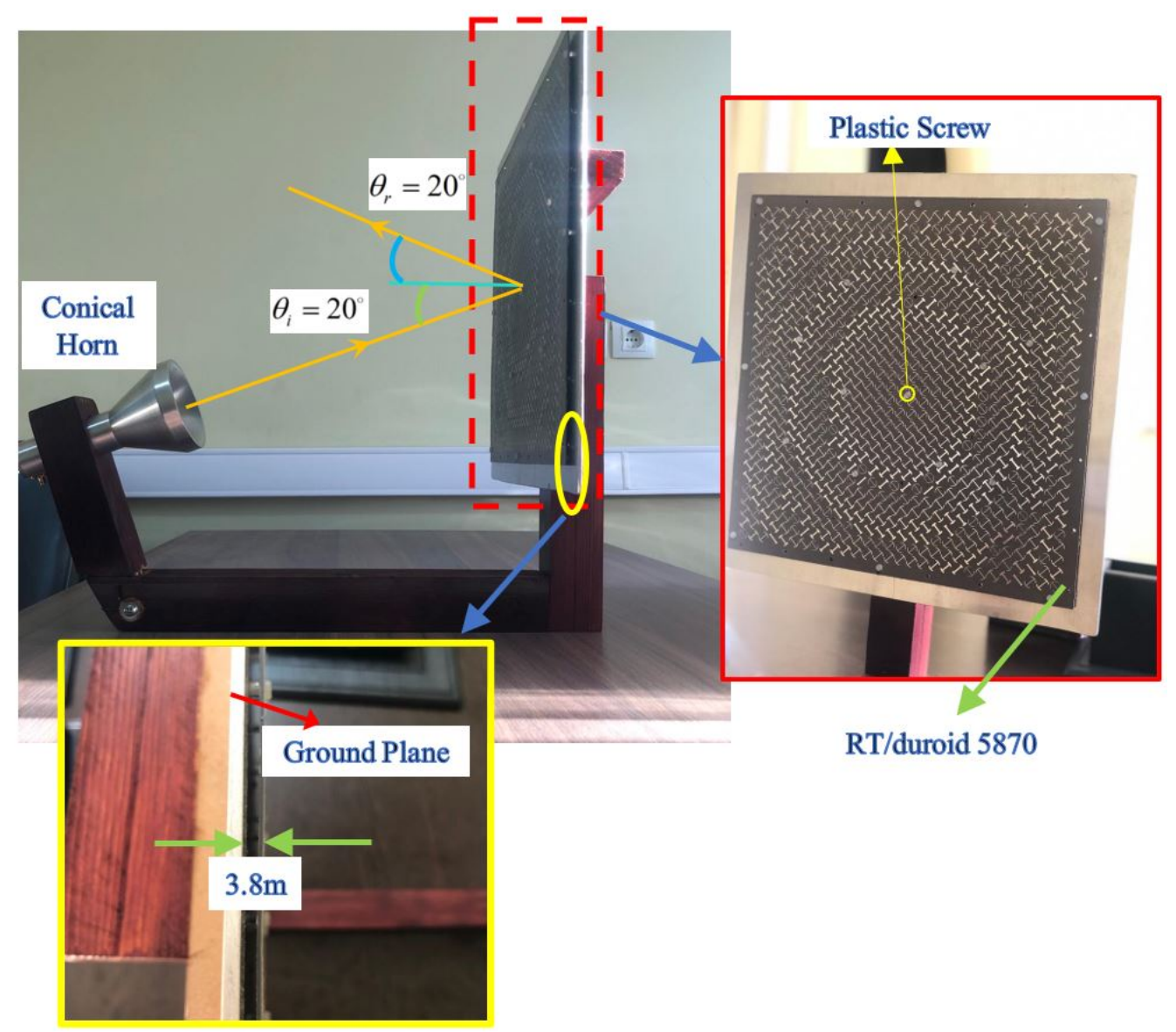

Figure 13. Fabrication of a prototype of metasurface and its position with respect to the horn antenna as the feeding system.

The simulation and measurement results for the co- and cross-polarization patterns on the E- and $\mathrm{H}$-plane are presented in Fig. 14 at two extreme frequencies (i.e, $14 \mathrm{GHz}$ and $19 \mathrm{GHz}$ ). It is clear that the sidelobe levels are below $-18 \mathrm{~dB}$ for all of the patterns in E-plane and below $-20 \mathrm{~dB}$ in $\mathrm{H}$-plane thanks to the use of a completely equiphase aperture. In addition, the cross-polarization levels are all below $-18 \mathrm{~dB}$, which are acceptable results.

\section{Method}

The measurement of the electromagnetic characteristics of the metasurface is carried out in two steps. First, the S-parameter and the pattern of the horn antenna are measured by a keysight vector network analyzer ENA E5080B series operating up to 20 $\mathrm{GHz}$. Second, the radiation pattern of the radiated beams from the metasurface is measured by this network. In order to measure the realized gain, the comparison technique is adopted by using two standard horn antennas working in $\mathrm{Ku}$ - and $\mathrm{K}$-bands.

\section{Discussion}

In order to benchmark the usefulness of the proposed broadening technique which is based on combining the TFPS method and PCT, a comparison is carried out between the frequency bandwidth of two metasurfaces which the first one is designed by only using PCT and the second one is designed using PCT+ TFPS method. The bandwidth and aperture frequency of the two configurations are shown in Fig. 15. As can be seen in Fig. 15, the 1-dB gain bandwidth of the first and the second metasurfaces are $17.47 \%$ (14.1-16.8 GHz) and 30.3\% (14-19 GHz), respectively. This result verifies the advantage of combining the PCT and TFPS method where an increment of nearly $13 \%$ is obtained in the bandwidth. The maximum aperture efficiency for the first and second metasurface is $60 \%$ and $63.62 \%$, respectively, which occur at $14.5 \mathrm{GHz}$ for both cases. As expected, three peaks are observed in the gain versus frequency plot. These peaks occur at $14.5 \mathrm{GHz}, 17 \mathrm{GHz}$, and $19 \mathrm{GHz}$ which are exactly 


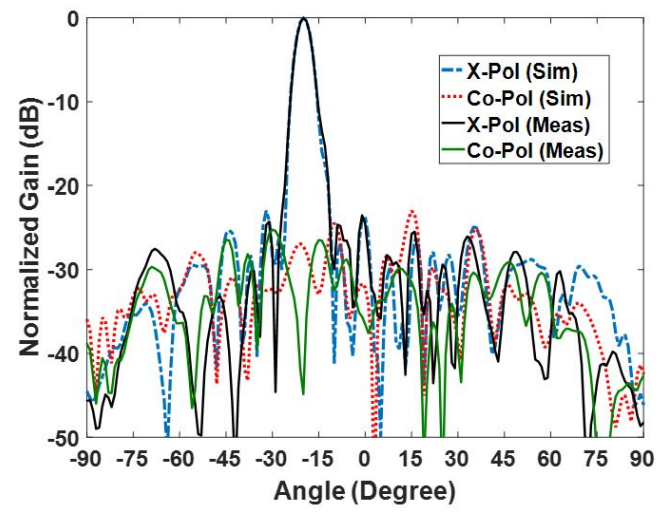

(a)

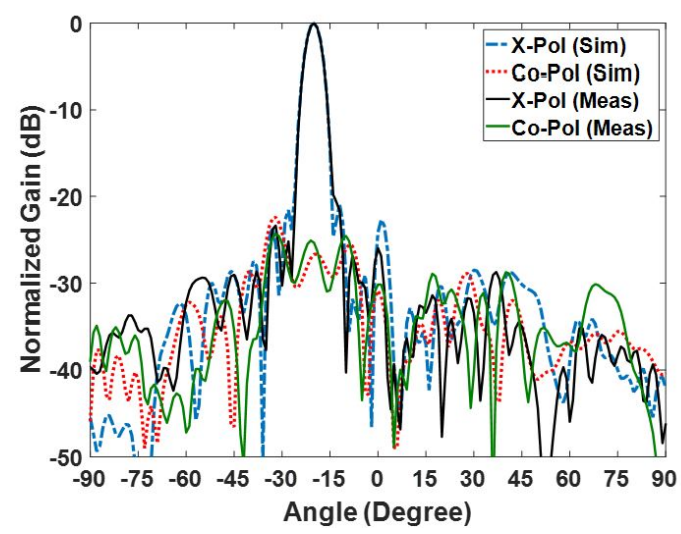

(c)

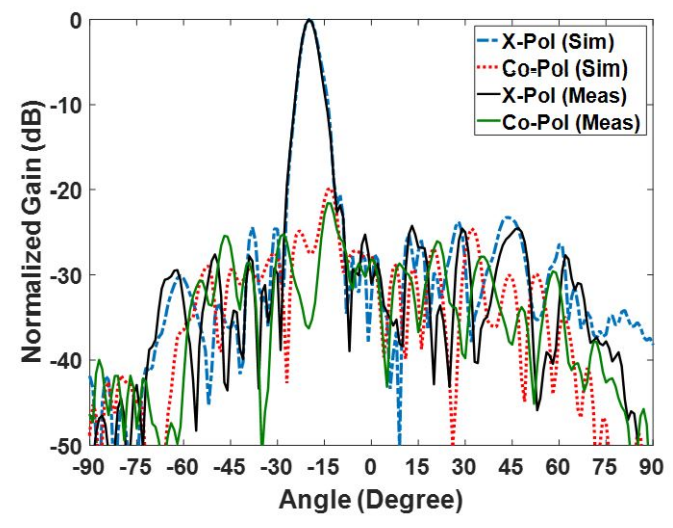

(e)

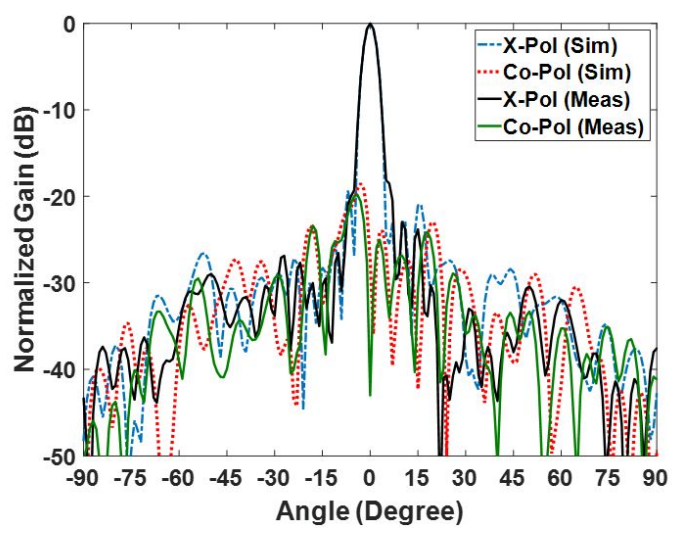

(b)

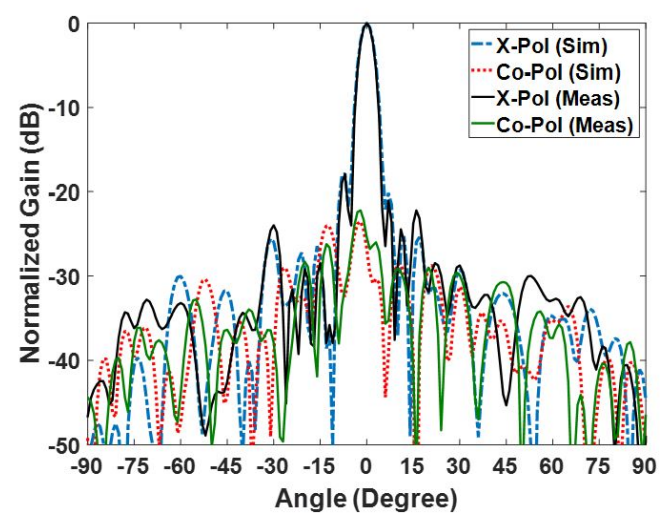

(d)

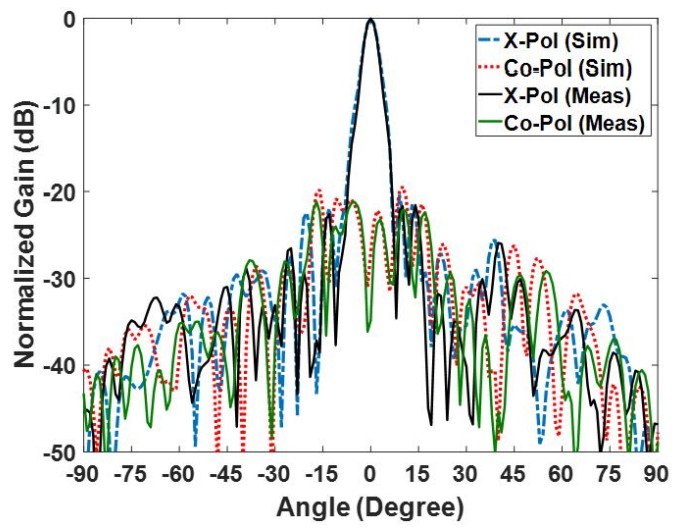

(f)

Figure 14. Comparision between the simulation and measurement results. (a) E-plane at $14 \mathrm{GHz}$, (b) H-plane at $14 \mathrm{GHz}$, (c) E-plane at $17 \mathrm{GHz}$, (d) H-plane at $17 \mathrm{GHz}$. (e) E-plane at $19 \mathrm{GHz}$. (f) H-plane at $19 \mathrm{GHz}$

alike with those are defined as the designed frequency in the TFPS method. As stated above, at the system design level, several optimizations are accomplished to find the best three frequencies which lead to the maximum 1-dB gain bandwidth. That is, the choice of three frequencies in $C F\left(f_{i}, \Delta \phi_{i}\right)$ effects on how the gain level varies in the whole band, and hence changes the 1-dB gain bandwidth.

In order to better highlight the effectiveness of the broadband technique introduced in this work, a comparison is done 


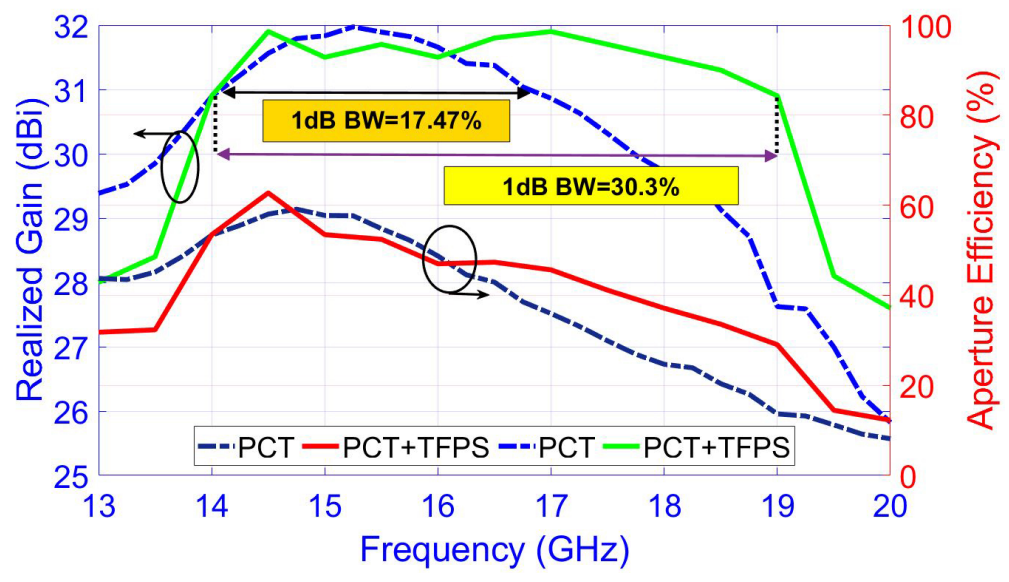

Figure 15. The comparisons between the bandwidth and aperture efficiency of the two metasurfaces which are developed by PRT and PCT+TFPS method. Noted that the measurement results of the metasurface developed by PCT+TFPS are compared with the simulation results of the metasurface developed by PCT.

\begin{tabular}{cccccccc}
\hline \hline Reference & Ref. $^{18}$ & Ref. $^{27}$ & Ref. $^{28}$ & Ref. $^{29}$ & Ref. $^{17}$ & Ref. $^{30}$ & This work \\
\hline Central frequency [GHz] & 20 & 9 & 10 & 10 & 10 & 8 & $\mathbf{1 5}$ \\
\hline Polarization & LP and CP & LP & LP & LP & LP & LP & LP \\
\hline Maximum aperture efficiency [\%] & 54.4 & 17.6 & NR & 48.2 & 20 & 55 & $\mathbf{6 4}$ \\
\hline Number of metal layers & 1 & 1 & 3 & 4 & 2 & 1 & $\mathbf{1}$ \\
\hline Element dimension [mm] & 7.5 & 7 & 6.5 & 15 & 6 & 18 & $\mathbf{1 0}$ \\
\hline Aperture dimension [mm] & $26 \lambda_{0}$ & $9 \lambda_{0}$ & $8.4 \lambda_{0}$ & $6.5 \lambda_{0}$ & $9.6 \lambda_{0}$ & $8 \lambda_{0}$ & $\mathbf{1 4 . 5} \lambda_{0}$ \\
\hline Maximum Gain [dBi] & 35.6 & 23 & 23 & 24 & 24.2 & 26.5 & $\mathbf{3 1 . 9}$ \\
\hline Side lobe level [dB] & $<-15$ & -15 & -10 & -17 & -13 & $-9^{\dagger},-13^{\dagger \dagger}$ & $-18^{\dagger},-22^{\dagger \dagger}$ \\
\hline F/D & 0.92 & 1 & 0.87 & 1.08 & 1 & 0.75 & $\mathbf{0 . 8 5}$ \\
\hline Bandwidth [\%] & 18.5 & 25 & $40^{*}$ & 18.6 & 24 & 35 & $\mathbf{3 0 . 3}$ \\
\hline Broadband techniques & PCT & $1-$ bit & TTD & PCT & PCT & TDFE & PRT+TFPS \\
\hline \hline
\end{tabular}

Table 2. The comparison among this work and previous works. The asterisks $\left(^{*}\right)$ in the bandwidth row denotes the 4-dB bandwidth. Other references report 1-dB gain bandwidth. In addition, TDFE stands for two degree of freedom elements and superscripts ${ }^{\dagger}$ and ${ }^{\dagger \dagger}$ denote the initial and end frequencies of the band. 'NR' stands for the not reported data.

between several works presented in the literature. These comparisons are observed in Table. 2. The comparisons show that the combination of the PCT and TFPS method leads to the improvement in 1-dB gain bandwidth, low side lobe level, low cross-polarization level, and aperture efficiency at the same time.

\section{References}

1. Nayeri, P., Yang, F. \& Elsherbeni, A. Z. Reflectarray antennas: theory, designs, and applications (John Wiley \& Sons, 2018).

2. Karimipour, M., Komjani, N. \& Aryanian, I. Shaping electromagnetic waves with flexible and continuous control of the beam directions using holography and convolution theorem. Sci. reports 9, 1-13 (2019).

3. Pozar, D. Wideband reflectarrays using artificial impedance surfaces. Electron. letters 43, 148-149 (2007).

4. Karimipour, M., Komjani, N. \& Aryanian, I. Broadband, dual-band reflectarray with dual orthogonal polarisation for single and multi-beam patterns. IET Microwaves, Antennas \& Propag. 13, 2037-2045 (2019).

5. Karimipour, M., Pirhadi, A. \& Ebrahimi, N. Accurate method for synthesis of shaped-beam non-uniform reflectarray antenna. IET Microwaves, Antennas \& Propag. 7, 1247-1253 (2013).

6. Karimipour, M. \& Komjani, N. Realization of multiple concurrent beams with independent circular polarizations by holographic reflectarray. IEEE Transactions on Antennas Propag. 66, 4627-4640 (2018). 
7. Mao, Y., Xu, S., Yang, F. \& Elsherbeni, A. Z. A novel phase synthesis approach for wideband reflectarray design. IEEE transactions on antennas propagation $\mathbf{6 3}, 4189-4193$ (2015).

8. Deng, R., Xu, S., Yang, F. \& Li, M. A single-layer high-efficiency wideband reflectarray using hybrid design approach. IEEE Antennas Wirel. Propag. Lett. 16, 884-887 (2016).

9. Bodur, H. \& Çimen, S. Reflectarray antenna design with double cutted ring element for x-band applications. Microw. Opt. Technol. Lett. 62, 3248-3254 (2020).

10. Guo, L., Yu, H., Che, W. \& Yang, W. A broadband reflectarray antenna using single-layer rectangular patches embedded with inverted l-shaped slots. IEEE Transactions on Antennas Propag. 67, 3132-3139 (2019).

11. Ning, P., Min, M., Guo, L. \& Feng, W. On the use of half-cut elements for single-layer wideband reflectarrays. IEEE Antennas Wirel. Propag. Lett. 20, 943-947 (2021).

12. Karimipour, M. \& Aryanian, I. Demonstration of broadband reflectarray using unit cells with spline-shaped geometry. IEEE Transactions on Antennas Propag. 67, 3831-3838 (2019).

13. Guo, W.-L., Wang, G.-M., Liu, K.-Y., Zhuang, Y.-Q. \& Ge, Q.-C. Design of single-layered ultrawideband high-efficiency circularly polarized reflectarray. IEEE Antennas Wirel. Propag. Lett. 17, 1386-1390 (2018).

14. Moustafa, L. et al. The phoenix cell: A new reflectarray cell with large bandwidth and rebirth capabilities. IEEE Antennas wireless propagation letters 10, 71-74 (2011).

15. Encinar, J. A. Design of two-layer printed reflectarrays using patches of variable size. IEEE Transactions on Antennas Propag. 49, 1403-1410 (2001).

16. Delafkar, H. \& Pirhadi, A. An accurate method for synthesis of reflecting elements to design wideband reflectarray antenna. IEEE Transactions on Antennas Propag. 66, 2392-2400 (2018).

17. Luyen, H., Yang, Z., Gao, M., Booske, J. H. \& Behdad, N. A wideband, single-layer reflectarray exploiting a polarization rotating unit cell. IEEE Transactions on Antennas Propag. 67, 872-883 (2018).

18. Zhang, Z., Luyen, H., Booske, J. H. \& Behdad, N. A dual-band, polarization-rotating reflectarray with independent phase control at each band. IEEE Transactions on Antennas Propag. (2021).

19. Zhang, X., Yang, F., Xu, S., Aziz, A. \& Li, M. Hybrid polarization-phase tuning methodology for reflectarray antennas. IEEE Transactions on Antennas Propag. (2021).

20. Feng, M. et al. Broadband polarization rotator based on multi-order plasmon resonances and high impedance surfaces. $J$. Appl. Phys. 114, 074508 (2013).

21. Hong-Ya, C. et al. Broadband perfect polarization conversion metasurfaces. Chin. Phys. B 24, 014201 (2015).

22. Zhang, L. et al. Adjustable wideband reflective converter based on cut-wire metasurface. J. Opt. 17, 105105 (2015).

23. Khan, M. I. et al. Multiband linear and circular polarization rotating metasurface based on multiple plasmonic resonances for c, $\mathrm{x}$ and $\mathrm{k}$ band applications. Sci. reports 10, 1-11 (2020).

24. Zhang, Z. et al. Single-layer metasurface for ultra-wideband polarization conversion: bandwidth extension via fano resonance. Sci. Reports 11, 1-8 (2021).

25. Yang, F., Deng, R., Xu, S. \& Li, M. Design and experiment of a near-zero-thickness high-gain transmit-reflect-array antenna using anisotropic metasurface. IEEE Transactions on Antennas Propag. 66, 2853-2861 (2018).

26. Yu, N. et al. Light propagation with phase discontinuities: generalized laws of reflection and refraction. science 334, 333-337 (2011).

27. Yu, S., Li, L. \& Kou, N. One-bit digital coding broadband reflectarray based on fuzzy phase control. IEEE Antennas Wirel. Propag. Lett. 16, 1524-1527 (2017).

28. Abadi, S. M. A. M. H., Ghaemi, K. \& Behdad, N. Ultra-wideband, true-time-delay reflectarray antennas using groundplane-backed, miniaturized-element frequency selective surfaces. IEEE Transactions on Antennas Propag. 63, 534-542 (2014).

29. Liu, S. \& Chen, Q. A wideband, multifunctional reflect-transmit-array antenna with polarization-dependent operation. IEEE Transactions on Antennas Propag. 69, 1383-1392 (2020).

30. Lia, X., Luo, Y., Wei, G. \& Yi, X. A novel single layer wideband reflectarray design using two degrees of freedom elements. IEEE Transactions on Antennas Propag. (2021). 


\section{Acknowledgements}

The authors would like to thank from Iran Telecommunication Research Center (ITRC) for providing the RF laminate and testing the prototype.

\section{Author contributions statement}

Dr. M. Karimipour proposed the main idea of this work. The simulations and writing the paper is also done by Dr. M. Karimipour. Dr. I. Aryanian conducted the experiments, analyzed the results, and review the paper. 